\title{
Editorial
}

\section{El transporte como una cuestión estructural}

El transporte en San Salvador se ha vuelto un grave y complicado problema, cuyas consecuencias sobrepasan su ámbito en cuanto tal. No se trata únicamente de poner orden en una actividad que se desenvuelve de la manera más errática y arbitraria posible. De ahí que los esfuerzos que el Viceministerio de Transporte viene haciendo para conseguir tal cosa desde hace algún tiempo arrojen unos resultados tan magros. El problema planteado por el transporte supera el desorden de tráfico vehicular al ser una de las actividades que más contribuye a la contaminación del medio ambiente y a deteriorar la calidad de vida en San Salvador y, por consiguiente, en el país entero.

No obstante la indiferencia y la resistencia de quienes hacen uso del transporte y de la población en general ante cambios necesarios y urgentes, el problema debe ser enfrentado, no sólo con determinación firme, sino también con ideas claras, viables y eficaces, y con cierta premura. Para ello se cuenta con una ciencia especializada en el tema, con una tecnología desarrollada y comprobada y con experiencia acumulada en muchos otros países del primer y del tercer mundo.

Ahora bien, un planteamiento realista del transporte debe pasar de las manifestaciones externas al análisis de su complejidad estructural. Las soluciones posibles no son fáciles, porque en la medida en que la cuestión del transporte sea planteado en términos estructurales, aquéllas también tendrán que serlo de manera obligada. El problema del transporte en San Salvador y, en general, en El Salvador, exige una solución integral; pero en la medida en que lo sea, ésta no estará al alcance del país mientras otros muchos problemas que lo aquejan no sean enfrentados de manera simultánea. 


\section{Un análisis estructural}

El transporte tiene una importancia singular para los habitantes de la zona metropolitana de San Salvador, donde se encuentra concentrada la mayor parte de la población del país. El 69 por ciento de los residentes en San Salvador, es decir, más de un millón de personas, utiliza el transporte colectivo diariamente para desplazarse de su vivienda al trabajo, para abastacerse de bienes y para acceder a los servicios indispensables. Toda esta población vive en una especie de cautividad, pues depende de la disponibilidad de este medio de transporte para desplazarse - un estimado de poco más de cuatro mil unidades motorizadas de muy diverso tamaño y cuya edad promedio oscila entre los dieciocho y los diez años, organizadas en unas 140 rutas, las cuales efectúan más de 26 mil recorridos diarios.

El trayecto de estas rutas se ha establecido gradualmente, en función del beneficio económico de su operador, es decir, conectando de forma casi exclusiva aquellos sitios donde se concentra la mayor cantidad de usuarios potenciales -centros donde tienen lugar actividades laborales, educativas, comerciales, deportivas, etc., y las terminales del transporte colectivo interdepartamental. En consecuencia, la mayoría de las rutas tienen como destino final el centro de San Salvador. Es así como el recorrido tiene forma de un anillo radial, cuya distancia promedio es de unos veintidós kilómetros. De ahí que los operadores hayan establecido terminales en el centro y en la periferia de la red, lo cual, por un lado, congestiona al primero y, por el otro, ha llevado a la apropiación, con frecuencia ilegal, de áreas públicas en los barrios residenciales; donde, además, son fuente de ruido, foco de suciedad y zona de mucha inseguridad para sus habitantes.

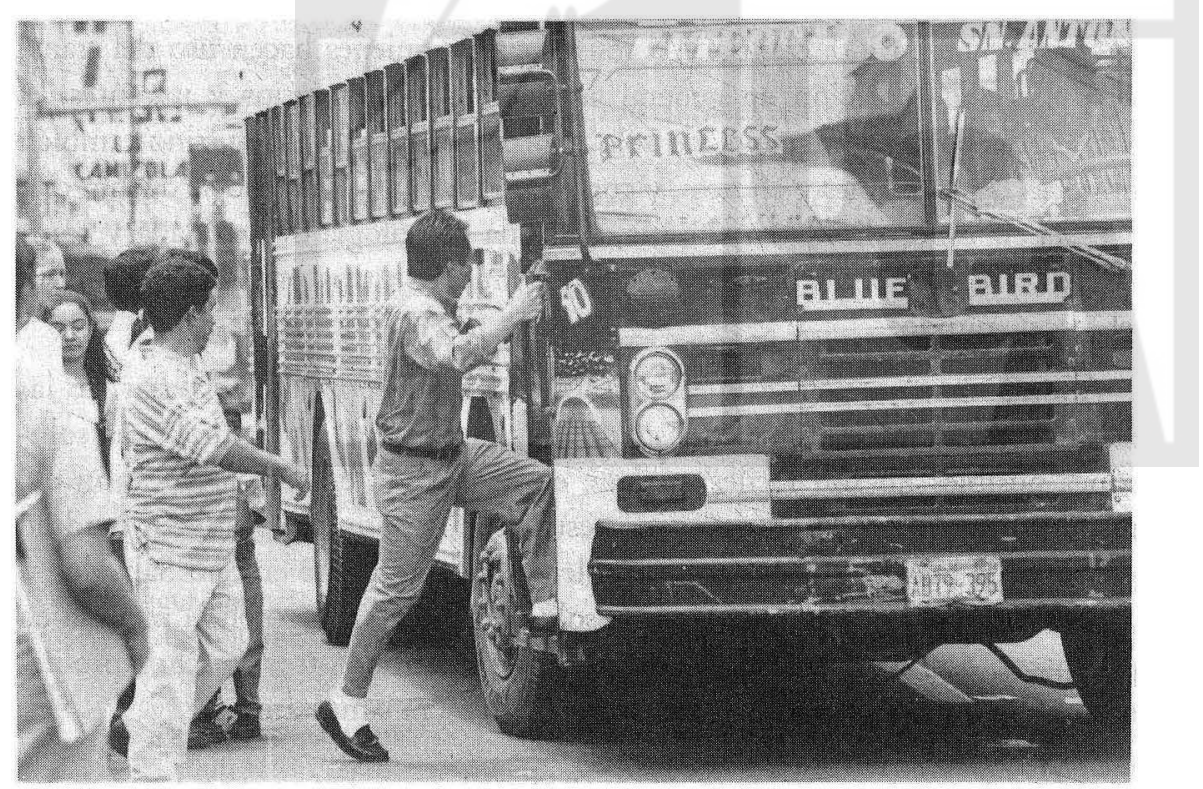


Las paradas a lo largo del trayecto de las rutas no han sido establecidas con un criterio funcional, sino que, en la práctica, el usuario, con la connivencia del conductor del vehículo, las ha ido fijando ahí donde más le conviene, es decir, en cualquier parte. Se ha llegado al extremo de que el usuario detiene el vehículo en mitad de la calle - con la anuencia del conductor, claro está. Las paradas son puntos de gran congestionamiento y de alto riesgo para los usuarios por la concentración de rutas en un mismo sitio y por la indisciplina de las unidades motorizadas, las cuales no hacen fila, no aguardan a que las que llegaron primero circulen, utilizan más de un carril y sólo continúan su recorrido cuando ya tienen dentro el número máximo de pasajeros, para lo cual utilizan cada vez con mayor frecuencia las bocacalles, obstaculizando la circulación y poniendo en grave riesgo la seguridad de otras personas.

Las frecuencias y los horarios de las unidades motorizadas no pueden ser definidos de forma racional porque se desconoce la demanda de usuarios. La inexistencia de control deja la velocidad de las unidades a discreción de sus conductores, quienes, por lo general, ignoran $\multimap$ sencillamente desconocensus límites racionales, lo cual es un factor más de inseguridad para el usuario y el peatón. El usuario no es informado sobre el recorrido de las rutas, sus paradas, su frecuencia y su horario. Esta información es transmitida de forma oral y es verificada por la costumbre. Las rutas aprobadas por el gobierno incluyen recorridos que implican maniobras que atentan contra la ordenación del tráfico, por ejemplo, las unidades hacen giros a la izquierda ahí donde no está permitido o en una vía de tres carriles se lanzan de un extremo al otro.

El transporte colectivo interurbano contribuye lo suyo al congestionamiento y al desorden general al mezclarse con el urbano, al utilizar las mismas paradas y al practicar los mismos vicios. En ninguna de las tres terminales interurbanas que sirven a San Salvador existe ordenamiento de entradas y salidas. Adicionalmente, El Salvador cuenta con una red ferroviaria subutilizada que, en la actualidad, podría ser muy útil para descongestionar la circulación de unidades motorizadas. Pero el ferrocarril fue abandonado a su suerte hasta el punto de dejarlo morir por falta de inversión y también de visión sobre sus potencialidades a mediano plazo. Las pocas unidades que todavía circulan lo hacen de manera lenta e insegura y, en tales condiciones, no pueden competir con las unidades motorizadas del transporte colectivo.

Los habitantes del área metropolitana de San Salvador experimentan dificultades serias para desplazarse, las cuales se deben al pésimo servicio que presta el transporte colectivo, a la inexistencia de medios alternativos no motorizados, a la ausencia de infraestructura y a la indiferencia de la sociedad. Todo ello se ve agravado por el crecimiento vegetativo de la población y el patrón del desarrollo urbano. Por razones diversas, que se discuten más adelante, San Salvador se ha configurado sin tener en cuenta la necesidad de desplazamiento de sus habitantes ni los medios para hacerlo, lo cual a su vez los obliga a 
ejecutar más movimientos y a demandar más servicio de transporte de lo estrictamente necesario. Si no se adoptan medidas eficaces pronto, la situación empeorará aún más. En poco tiempo, el 50 por ciento de la población salvadoreña residirá en zonas urbanas y siendo el transporte motorizado la actividad que más energía consume y también la que más contamina la atmósfera, los recursos no renovables se agotarán más rápido y la contaminación será aún mayor.

La elevada frecuencia de desplazamientos y la congestión derivada de ella son causa de una degradación medioambiental severa. El 75 por ciento de la contaminación atmosférica en ciudades como San Salvador es generada por los motores y los combustibles del tráfico vehicular - el resto se debe a la emisión de gases industriales. La emisión de humos en las proximidades de las concentraciones humanas pone en grave peligro la salud pública de quienes residen en ellas, la niebla fotoquímica (mezcla de gases y partículas oxidadas por el sol) es una amenaza para la salud pública regional. la lluvia ácida (dióxido de azufre, óxidos de nitrógeno e hidrocarburos) deteriora la foresta y degrada la calidad del suelo, las concentraciones atmosféricas de gases que causan el efecto invernadero (dióxido de carbono, metano, óxidos de nitrógeno, clorofluorocarbonos y ozono troposférico) ocasionan cambios climáticos con consecuencias desastrosas para la población.

El congestionamiento, que se agrava por cualquier percance que ocurra en las vías, significa más gasto de energía, más contaminación, más tiempo y mayor estrés, cuya manifestación más palpable y frecuente es la agresión verbal y a veces física —en San Salvador se asesina por el derecho de vía: conductores fuera de sí agreden a balazos a quien les reclamó una maniobra imprudente u obstruir la vía. Así, pues, además de la degradación de la atmósfera por la emisión de gases, el consumo energético aumenta de una manera evitable, el nivel del ruido es elevado, la frecuencia de accidentes es mayor y el espacio público es ocupado de una manera excesiva. Es obvio, entonces, que el transporte motorizado es una de las causas más determinantes del deterioro de la calidad de vida y del medio ambiente no sólo local, sino también regional y global.

El trato inhumano que el usuario del transporte colectivo, el peatón y el ciclista reciben de los conductores, la importación no controlada de vehículos, el mal estado de los motores, la disposición inadecuada de los desechos, los combustibles y los lubricantes de las unidades motorizadas, la inadecuación de la infraestructura vial y la existencia de mafias y del tráfico de influencias agravan una situación que, de por sí, ya es bastante crítica.

Los habitantes del área metropolitana de San Salvador experimentan dificultades serias para desplazarse, las cuales se deben al pésimo servicio que presta el transporte colectivo, a la inexistencia de medios alternativos no motorizados, a la ausencia de infraestructura y a la indiferencia de la sociedad. 


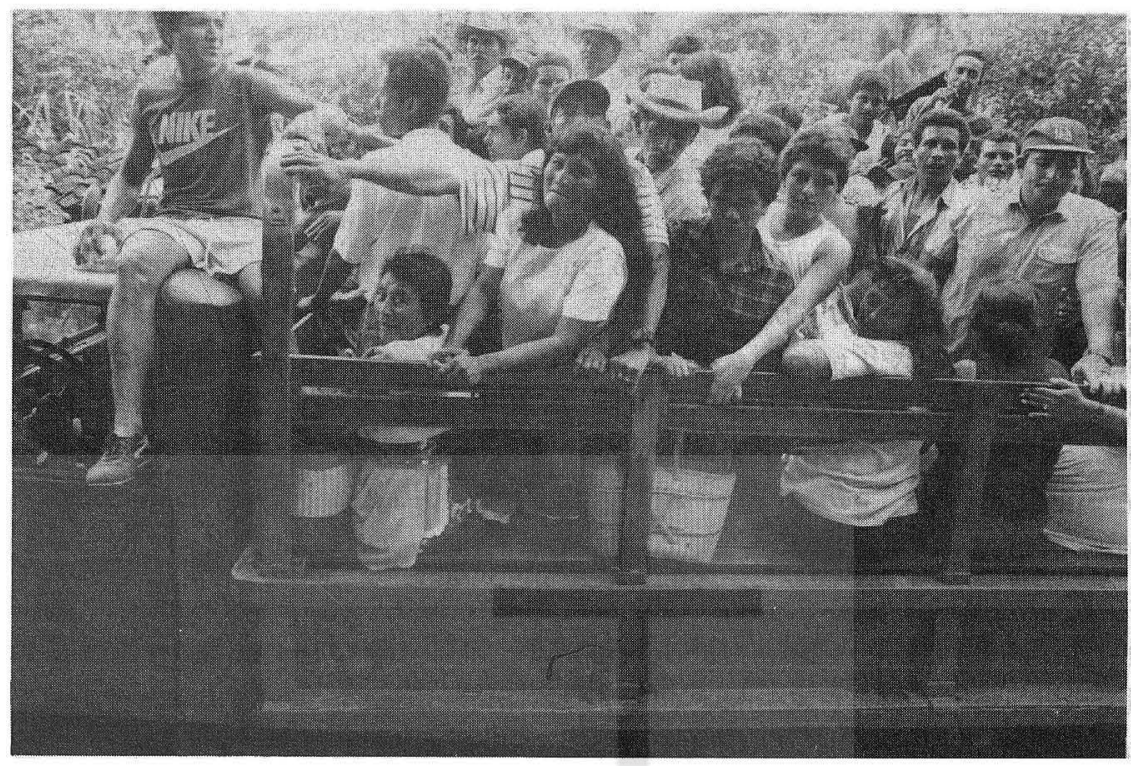

En conclusión, el sistema de transporte no es sostenible desde la perspectiva social, porque no garantiza el establecimiento y la conservación de las relaciones humanas, la satisfacción de las necesidades básicas de los ciudadanos, ni su seguridad; ni desde la perspectiva ecológica, por su elevado consumo energérico y su elevada contaminación del medio ambiente. La frustración de la población consciente del mal servicio del transporte colectivo y las batallas del Viceministerio de Transporte son reflejo de esta inviabilidad, aunque muy probablemente ni la una ni el otro sean del todo conscientes de sus implicaciones estructurales. El problema no está tanto en la cantidad de usuarios del transporte colectivo, sino en la mala calidad del servicio ofrecido, en sentido amplio, pero no por eso menos estricto. En suma, el estado del transporte es un reflejo del subdesarrollo generalizado de El Salvador.

\section{Ciudad y transporte: una realidad estructural}

El estado deplorable del transporte está estrechamente relacionado con la estructura de la ciudad. La estructura de San Salvador y su sistema de transporte se determinan mutuamente. La ciudad se ha ido estructurando de tal manera que su sistema de transporte, el cual pareçe tan natural, es, en realidad, una imposición de dicha estructuración. Pero también el desarrollo del sistema de transporte, tal como existe en la actualidad, ha impulsado e incluso permitido que San Salvador haya ido adquiriendo la forma que hoy posee.

Por una mezcla de indiferencia e ignorancia, San Salvador es una ciudad muy dispersa. Sus centros de actividad se encuentran diseminados "espontáneamente" a lo largo y ancho de su superficie. En efecto, la ciudad posee unos cuantos puntos céntricos, distribuidos sobre su territorio sin mayor orden, en los cuales tiene lugar su actividad productiva, comercial, política, administra- 
tiva, educativa, social, etc. Esta dispersión espacial da paso a otra de carácter social. Las ciudades dormitorio que la rodean son un buen ejemplo de esta dispersión funcional. Los habitantes que duermen en ellas están obligados a hacer largos desplazamientos para desarrollar sus actividades cotidianas o simplemente para relacionarse con los demás. Dada la estructura de San Salvador y del transporte, esos desplazamientos sólo pueden ser motorizados. Esto es así porque su centro segregó espacial y socialmente las áreas residenciales de las actividades secundarias y terciarias. Las nuevas tecnologías del transporte influyeron de forma determinante en la configuración de esta realidad territorial y social urbana dispersa. Es indudable que San Salvador tiene casi todo aquello que se puede esperar encontrar en una ciudad promedio del tercer mundo, pero muy disperso.

El sistema de transporte es el encargado de unir estos espacios y en virtud de ello se ha convertido en el soporte clave de la estructura de la ciudad. Esta distribución tan poco racional de las actividades aumentó la cantidad de desplazamientos motorizados, la longitud de las distancias a recorrer y los tiempos necesarios para hacerlo. Para empeorar las cosas, el medio privilegiado a través del cual se concreta esta unificación espacial y social es el auto privado, que, paradójicamente, es el que más energía emplea y el que más contamina el medio ambiente. Por otro lado, la concentración de actividades en unos cuantos puntos congestiona el espacio y, en consecuencia, eleva los costos económicos, sociales y medio ambientales de la vida ciudadana.

... el sistema de transporte no es sostenible desde la perspectiva social, porque no garantiza el establecimiento y la conservación de las relaciones humanas, la satisfacción de las necesidades básicas de los ciudadanos, ni su seguridad; ni desde la perspectiva ecológica, por su elevado consumo energético y su elevada contaminación del medio ambiente.

Naturalmente, el que se desplaza busca disminuir los costos e inconvenientes de sus movimientos y por eso recurre al auto privado. Las ventajas inmediatas de este medio privilegiado son innegables, pero no es lo más racional desde una perspectiva social y medio ambiental. El predominio del uso de este medio, además de la consideración económica, tiene otra de carácter cultural. En la sociedad salvadoreña predomina lo que bien podría ser llamada la cultura del auto privado. Su predominio es tal que muchas personas no se desplazan, aunque lo deseen o necesiten, si no disponen de él. El vehículo privado, por influencia directa de la cultura estadounidense, proporciona seguridad, prestigio y estatuto social a su propietario. 
El transporte, a su vez, ha determinado el crecimiento y la configuración de la ciudad. La disminución del tiempo empleado en el recorrido, gracias al desarrollo tecnológico, ha estimulado la dispersión de los centros de actividad ciudadana. El desarrollo de la infraestructura y la tecnología contribuye, de manera simultánea, a la creación de nuevos centros de gravedad y a su dispersión, puesto que dondequiera que se coloquen, siempre serán accesibles para la población. En la práctica, no hay ningún obstáculo que impida la multiplicación y la dispersión de los centros de actividad urbana, aunque para ello se ocupe cada vez más territorio. La infraestructura del transporte y el crecimiento descontrolado del parque motorizado han roto los límites de la ciudad de San Salvador al hacer que todos los sitios sean accesibles.

No obstante la expansión errática de la ciudad, su configuración dispersa responde a un concepto que estuvo muy en boga, entre los urbanistas de hace tan sólo unas cuantas décadas. La dispersión de los centros de actividad pretendía responder al desafío planteado por el rápido crecimiento de las ciudades. En realidad, era una forma de diluir la complejidad de la urbanización en expansión acelerada a costa del territorio disponible. Los especialistas pensaron que la mejor solución era crear una ciudad funcional, cuyos sectores estuvieran delimitados de forma muy clara y cuya unidad espacial y social fuera dada por el sistema del transporte. Es así como las funciones de la ciudad se segregaron, pero a base de consumir energía y recursos naturales de toda clase.

En esta ciudad funcional, la clave estaba en las redes y en la disposición de un buen acceso a las mismas. En consecuencia, fueron surgiendo grandes infraestructuras viarias y, en esto, San Salvador no ha sido la excepción. Pero, contrario a lo que pudiera esperarse, las redes no fomentaron una mayor y mejor comunicación, sino que más bien aislaron a los ciudadanos. Las interacciones entre éstos se volvieron más difíciles, mientras que los desplazamientos, forzados por la dispersión de la estructura urbana, aumentaron, generando un gasto energético enorme. El uso de medios motorizados para el desplazamiento, en particular el auto privado, se impuso de manera inexorable.

Esta modalidad urbana, tal como se ha visto arriba, presupone la ocupación y el acceso al suelo, puesto que la dispersión de los centros de actividad sólo puede tener lugar sobre un suelo aparentemente ilimitado. Su localización es relativamente irrelevante, porque el acceso a ellos es posible gracias a la existencia de infraestructura, equipo y sistemas de transporte, cuya disponibilidad también parece no tener límite. Todo esto se combina para hacer que los desplazamientos lleguen a su destino con mayor o menor facilidad. Para el usuario del transporte colectivo, significa proximidad de su vivienda a los centros donde tienen lugar las actividades que constituyen la trama de su vida; para quien dispone de auto privado, significa disponibilidad de aparcamiento y para el peatón y el ciclista, posibilidad real para moverse. El acceso también 
determina la calidad, la atracción y el valor del suelo. Su uso y explotación aumentan o disminuyen la relevancia de estos tres elementos. De ahí que la localización de los centros de actividad y, por consiguiente, la configuración del sistema del transporte varíen de acuerdo a estos factores y no tanto por motivaciones de carácter humanitario, solidario o medio ambiental.

Es inevitable que una ciudad como San Salvador, cuya estructura fundamental descansa en la utilización extensiva del suelo, deteriore de manera amenazante el medio ambiente. El uso y la explotación de la tierra por parte de la ciudad ha ido destruyendo el suelo agrícola y forestal, mientras que la ampliación de la red vial que los acompaña ha ido desestructurando los ecosistemas, al colocar más carga de la que pueden soportar - la disponibilidad de agua potable se ha vuelto cada vez más crítica. En la misma medida en que el espacio público se ha ido ocupando, el tráfico motorizado masivo se ha ido convirtiendo en una de las peores amenazas para la vida ciudadana. La emisión de gases, el nivel del ruido, la cantidad de accidentes, el tiempo que toman los desplazamientos y la desestructuración de los sistemas naturales han hecho insoportable la vida en San Salvador y sus alrededores, en las últimas dos décadas.

La dinámica del consumo que todo lo vuelve obsoleto con asombrosa rapidez refuerza esta tendencia a explotar y desestructurar cada vez más intensamente el entorno de la ciudad. Esta realidad es la que se encuentra en el fondo de la polémica sobre el destino de la finca El Espino y la cordillera del Bálsamo. Las empresas dedicadas a la construcción de urbanizaciones sólo consideran sus ganancias, no importándoles las consecuencias sociales y ecológicas de la explotación sin medida del suelo. En esto, el capitalismo es simplista y cortoplacista.

Dentro de este horizonte, la política del transporte debe ir mucho más allá de su ordenamiento o de impedir la conducción de vehículos en estado alcohólico o drogado. La prioridad debe ser mejorar la calidad del medio ambiente, es decir, el sistema del transporte debe ser ecológicamente sostenible.

\section{Propuesta estructural: la sostenibilidad urbana}

El San Salvador actual es fiel reflejo de la sociedad capitalista con esa peculiaridad tan suya de ocasionar más males que bienes, por lo menos para la mayor parte de sus habitantes. La ciudad capital muestra la peor cara de la libertad y del individualismo, del consumo y del afán de lucro. Ahora bien, la determinación estructural de la ciudad y su sistema de transporte vincula intrínsecamente cualquier respuesta que intente darse a ambas problemáticas. 
determina la calidad, la atracción y el valor del suelo. Su uso y explotación aumentan o disminuyen la relevancia de estos tres elementos. De ahí que la localización de los centros de actividad y, por consiguiente, la configuración del sistema del transporte varíen de acuerdo a estos factores y no tanto por motivaciones de carácter humanitario, solidario o medio ambiental.

Es inevitable que una ciudad como San Salvador, cuya estructura fundamental descansa en la utilización extensiva del suelo, deteriore de manera amenazante el medio ambiente. El uso y la explotación de la tierra por parte de la ciudad ha ido destruyendo el suelo agrícola y forestal, mientras que la ampliación de la red vial que los acompaña ha ido desestructurando los ecosistemas, al colocar más carga de la que pueden soportar — la disponibilidad de agua potable se ha vuelto cada vez más crítica. En la misma medida en que el espacio público se ha ido ocupando, el tráfico motorizado masivo se ha ido convirtiendo en una de las peores amenazas para la vida ciudadana. La emisión de gases, el nivel del ruido, la cantidad de accidentes, el tiempo que toman los desplazamientos y la desestructuración de los sistemas naturales han hecho insoportable la vida en San Salvador y sus alrededores, en las últimas dos décadas.

La dinámica del consumo que todo lo vuelve obsoleto con asombrosa rapidez refuerza esta tendencia a explotar y desestructurar cada vez más intensamente el entorno de la ciudad. Esta realidad es la que se encuentra en el fondo de la polémica sobre el destino de la finca El Espino y la cordillera del Bálsamo. Las empresas dedicadas a la construcción de urbanizaciones sólo consideran sus ganancias, no importándoles las consecuencias sociales y ecológicas de la explotación sin medida del suelo. En esto, el capitalismo es simplista y cortoplacista.

Dentro de este horizonte, la política del transporte debe ir mucho más allá de su ordenamiento o de impedir la conducción de vehículos en estado alcohólico o drogado. La prioridad debe ser mejorar la calidad del medio ambiente, es decir, el sistema del transporte debe ser ecológicamente sostenible.

\section{Propuesta estructural: la sostenibilidad urbana}

El San Salvador actual es fiel reflejo de la sociedad capitalista con esa peculiaridad tan suya de ocasionar más males que bienes, por lo menos para la mayor parte de sus habitantes. La ciudad capital muestra la peor cara de la libertad y del individualismo, del consumo y del afán de lucro. Ahora bien, la determinación estructural de la ciudad y su sistema de transporte vincula intrínsecamente cualquier respuesta que intente darse a ambas problemáticas. 


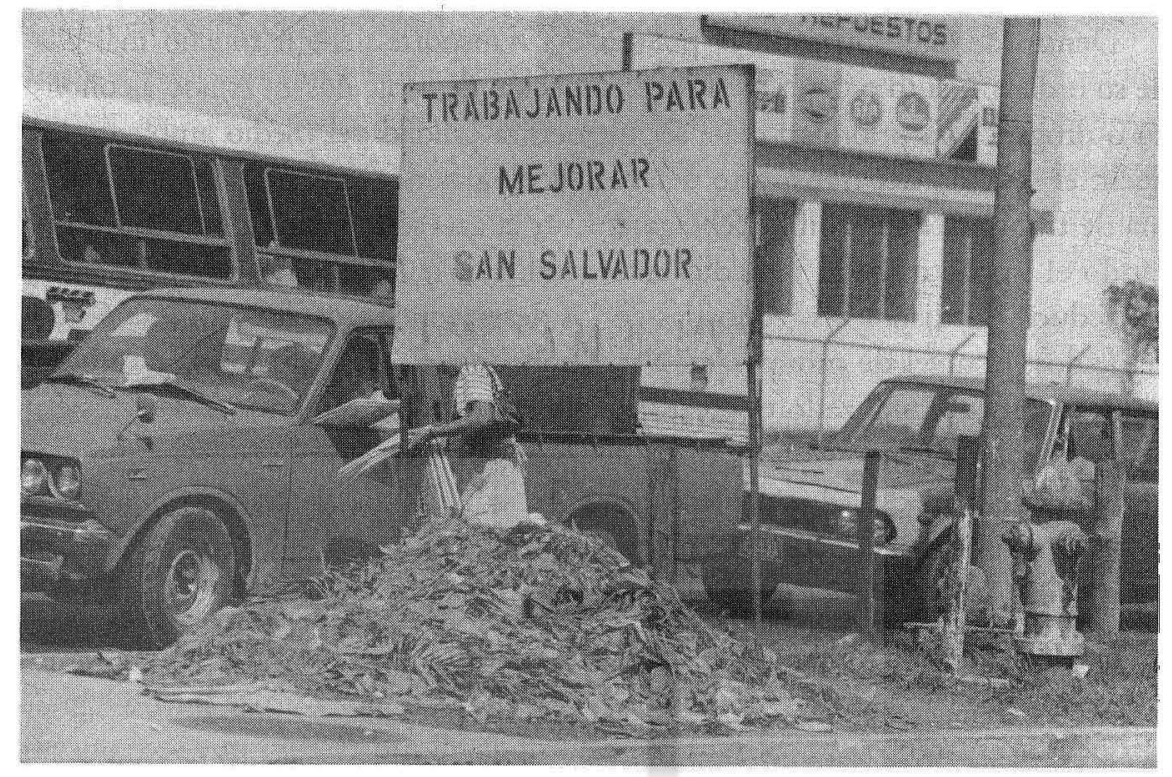

Sin embargo, de la misma manera que San Salvador concentra los graves problemas sociales $y$ medio ambientales del país, es también el lugar para encontrar una solución, incluyendo la participación de sus habitantes. El objetivo último de cualquier respuesta real consiste en mejorar las condiciones de vida de sus habitantes, haciendo de San Salvador una ciudad más habitable y acogedora.

No se parte de cero en esta empresa, puesto que ya existe tecnología y experiencia. San Salvador misma cuenta con algunos estudios buenos sobre las alternativas recomendables. En ellos, una cosa queda clara: la red radial actual es obsoleta desde el punto de vista de la eficacia del desplazamiento y mortalmente destructiva desde la perspectiva del medio ambiente. Esta clase de redes sólo es eficaz en las poblaciones pequeñas, donde el congestionamiento es relativamente bajo, pero no en una concentración urbana tan extensa como la zona metropolitana de San Salvador.

La dificultad mayor para iniciar los cambios recomendados estriba en que la vida ciudadana sólo puede mejorar si se alteran los patrones de producción y consumo. Por lo que toca al transporte $-\mathrm{y}$ a cualquier otra clase de consumo masivo-, esto implica reducciones drásticas en el uso de energías no renovables y materias primas y en conservar la cantidad de residuos y poluyentes dentro de las posibilidades de compensación del medio ambiente natural. No existe otra forma para crear alternativas que superen los desafíos planteados en la actualidad, sin comprometer el futuro de las próximas generaciones, en cuanto a la calidad del medio ambiente, el consumo de energías no renovables y la generación de residuos. 
Dentro de este horizonte, la política del transporte debe ir mucho más allá de su ordenamiento o de impedir la conducción de vehículos en estado alcohólico o drogado. La prioridad debe ser mejorar la calidad del medio ambiente, es decir, el sistema del transporte debe ser ecológicamente sostenible. Un sistema de transporte sostenible debe garantizar el acceso al mismo, pero minimizando el impacto social y medio ambiental. Por consiguiente, es indispensable reducir las distancias recorridas en los desplazamientos, la importación de vehículos, el nivel de motorización y el consumo de combustibles - aumentando el uso de catalizadores efectivos-, porque el incremento de la emisión de gases y de la generación de otros residuos está relacionado con el aumento de la circulación de vehículos motorizados, con las distancias recorridas por éstos, con su cantidad, sus características y su velocidad.

Estas reducciones sólo son posibles mediante la sustitución del transporte privado, el que mayor impacto medio ambiental ocasiona, por el transporte colectivo, al cual, además, todo ciudadano tiene derecho. Esto se puede lograr ofreciendo un transporte colectivo de calidad al mismo tiempo que se reducen los incentivos para el transporte privado - espacio para aparcar, apertura de nuevos carriles, mejora de los existentes, etc.- y se penaliza fiscalmente su uso - y no su propiedad. No se puede esperar que el tráfico se modere, es decir, que la cantidad de unidades motorizadas disminuya así como también su velocidad, si de forma simultánea se incentiva el uso del transporte privado y no se crean alternativas para el transporte colectivo y no motorizado. En cuanto a este último, es indispensable desarrollar la infraestructa para que el peatón y el ciclista circulen con seguridad y ya no sean considerados como intrusos por los usuarios del medio motorizado.

La oposición tenaz que los empresarios del transporte colectivo hacen en la actualidad a las tímidas reformas que el Viceministerio de Transporte impulsa, presagia la dura lucha que habrá que librar para llevar

a cabo una transformación no sólo del transporte, sino de la ciudad misma de San Salvador si, en realidad, existe voluntad para que la vida en ella sea vivible y acogedora.

A corto plazo es posible redistribuir el flujo del tráfico vehicular, urilizando las vías menos congestionadas; abrir las vías cerradas por el comercio informal; controlar los estacionamientos; priorizar el transporte público sobre el privado; diseñar y construir áreas peatonales, para ciclistas y para el comercio informal; limitar la cantidad de autos privados; renovar la flota del transporte colectivo y tal vez prohibir la circulación de microbuses; descen- 
tralizar el comercio informal; reconsiderar las rutas del transporte colectivo, descongestionando las vías y canalizándolo en vías paralelas; suprimir las paradas arbitrarias; aplicar con rigor el reglamento de tránsito y las sanciones previstas por su violación, sobre todo a los conductores de las unidades del transporte colectivo; impulsar alternativas que utilicen energías renovables no contaminantes, etc.

Estas limitaciones por sí solas no bastan, sino que deben ir acompañadas por programas ambientales, orientados a buscar energía alternativa, estudios del impacto de la infraestructura en el medio ambiente, análisis del acceso y la conducta del usuario del transporte y la transformación de la mentalidad de la población en general.

Aunque aparentemente estas propuestas parecen inalcanzables, no lo son si se analizan con cuidado. Si los sitios donde los habitantes desarrollan la mayoría de sus actividades cotidianas se aproximan a su lugar de residencia, los desplazamientos motorizados y sus repercusiones negativas necesariamente tendrían que reducirse de una manera relevante. Simultáneamente, la nueva estructura urbana hará que el uso del transporte privado pase de ser una necesidad impuesta a ser una opción. Es una imposición en cuanto el ciudadano no tiene alternativa, dada la estructuración de la ciudad y el estado de su sistema de transporte; pero se vuelve una opción cuando cuenta con una alternativa de calidad en el servicio colectivo.

Así, pues, la potenciación del transporte público es crucial para que el desplazamiento urbano adquiera su dimensión social y medio ambiental real. Las ventajas de este planteamiento son indiscutibles en cuanto ahorro energético, utilización racional del suelo disponible, reducción del congestionamiento, disminución del nivel de ruido, aumento de la seguridad urbana, además de otros beneficios. Las dificultades de una política orientada a la creación de un transporte sostenible son dos. El primer obstáculo que hay que superar es la costumbre a recurrir al transporte privado, como si ésta fuera la única alternativa posible. Es necesario que la menta-

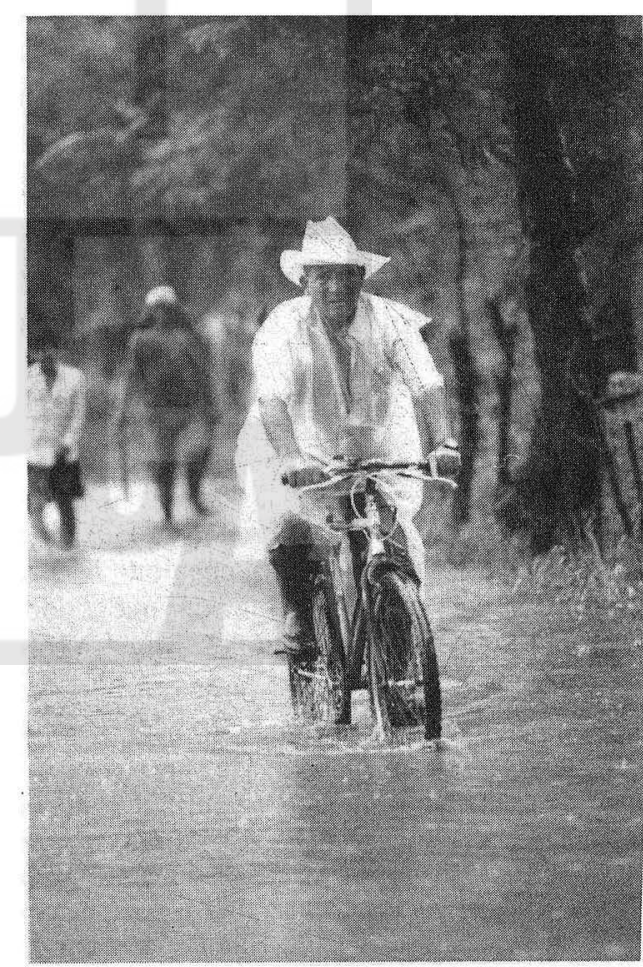


lidad siudadana se haga cargo de la necesidad de ahorrar energía y de usar los recursos naturales de una manera inteligente, lo cual supone un cambio cultural no exento de dificultades. El segundo obstáculo es la transformación necesaria de las estructuras económicas y sociales, lo cual entraña otra serie de dificultades no menos graves que el cambio de patrones culturales introyectados durante décadas.

La oposición tenaz que los empresarios del transporte colectivo hacen en la actualidad a las tímidas reformas que el Viceministerio de Transporte impulsa, presagia la dura lucha que habrá que librar para llevar a cabo una transformación no sólo del transporte, sino de la ciudad misma de San Salvador si, en realidad, existe voluntad para que la vida en ella sea vivible y acogedora. Los costos económicos, políticos y sociales serán elevados, pero los beneficios que se obtendrían de una reforma de tal envergadura serían mucho mayores. Aparte de no haber otra alternativa a largo plazo. El cambio es, de hecho, una imposición de la baja calidad de la vida urbana en el gran San Salvador de finales del segundo milenio. El supuesto fundamental es el compromiso con la vida y el bienestar de los ciudadanos de la capital.

San Salvador, 26 de agosto de 1999. 